\title{
Fault Detection and Isolation for Nonlinear System via ESO
}

\author{
Maryam Naghdi \\ Department of Electrical and \\ Robotics Engineering, \\ Shahrood University, \\ Shahrood, Iran.
}

\author{
Mohamad Ali Sadrnia \\ Department of Electrical and \\ Robotics Engineering, \\ Shahrood University \\ Shahrood, Iran.
}

\author{
Javad Askari \\ Department of Electrical and \\ Computer Engineering, Isfahan \\ University of Technology \\ Isfahan, Iran
}

\begin{abstract}
In this paper, a fault detection and isolation system for nonlinear systems is presented. Fault detection and isolation is accomplished by using extended state observer (ESO) and fuzzy logic system. The major of observer-based fault detection methods rely on the accurate mathematical model of the system, but in the real world an accurate model of the system may not be available. The ESO is different from conventional observers, it does not require an accurate model of the system, it provides vital information for fault detection with only partial information of the plant. The ESO has ability to augment unknown dynamics combined with unknown external disturbance as extended state and estimate it in real time by using given input-output data. This paper presents a new sensor fault detection and isolation (FDI) via ESO and fuzzy logic system. A two-tank system is used as a case study. The simulation results confirm the simplicity and effectiveness of the proposed FDI technique.
\end{abstract}

\section{Keywords}

Fault detection and isolation, Nonlinear system, Extended state observer, Fuzzy logic system.

\section{INTRODUCTION}

Due to the existence of nonlinearities in engineering systems, fault diagnosis of nonlinear systems much more have been studied in recent years. One of the most powerful approaches for fault detection is the model-based approach [1]. The presence of faults is detected by means of the residuals, the residuals are difference between the system outputs and model outputs. They are ideally zero, and when the fault occurs they become non-zero [2-4]. The common model-based analytical redundancy approaches are [5] diagnostic observers, parity space, parameter estimation approaches. Parity space approach is constructed on the linear system theories, which cannot easily be developed to nonlinear cases. Parameter estimation approach depends on the accurate dynamic models of the process [1].

Among model-based analytical redundancy methods, observer-based approaches is the most popular way to achieve high-performance fault diagnosis [4,6-8]. However, the model-based fault detection requires the accurate model of the monitored system [9]; but in the real world the accurate system model is often unavailable. The general conceptual structure of model-based fault detection is shown in Figure $1[9]$.

The extended state observer introduced in this paper is different from conventional observers, such as unknown input observer, disturbance observer and generalized observer; it does not require an accurate model of the system. The ESO requires little knowledge of the plant model, such as the order of the system. Because it does not use a model, it is simpler and easier to construct. It is also capable of filtering the measurement noise.

In this paper, firstly the residuals are generated by ESO without detailed knowledge about the dynamic system.

In fact, due to the measurement noise, external disturbance and modeling uncertainty, the residuals are never zero, even if there is no fault. The fuzzy logic system is a precise approach for handling uncertainties. Therefore, the fuzzy system can be applied to evaluate the residuals.

This paper is organized as follows. Section 2 describes the design of ESO. Section 3 describes the two-tank system. Section 4 presents sensor fault detection. Section 5 presents fault isolation. Section 6 gives conclusions about the presented technique.

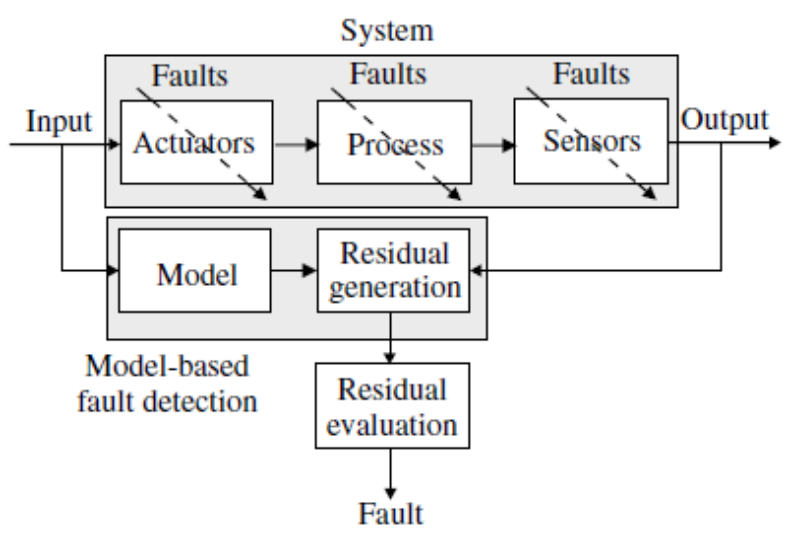

Fig. 1. General conceptual scheme of fault detection

\section{ESO}

The concept of ESO proposed by Han [10] as follows. Consider a nonlinear system that described by:

$$
y^{(n)}=f(y, \dot{y}, \ldots, w(t))+b u
$$

Then, a state space description of (1) is: 


$$
\left\{\begin{array}{l}
\dot{x}_{1}=x_{2} \\
\vdots \\
\dot{x}_{n}=f\left(x_{1}, \ldots, x_{n}, w(t)\right)+b u \\
y=x_{1}
\end{array}\right.
$$

where $f\left(x_{1}, \ldots, x_{n}, w\right)$ is nonlinear functions, $u$ is the control input, $y$ is the system output, $x_{1}, x_{2}, \ldots, x_{n}$ are system states, $w(t)$ is the external disturbance and $b$ is a constant.

According to ESO theory, we define $x_{n+1}=f\left(x_{1}, \ldots, x_{n}, w\right)$ and the new state space is:

$$
\left\{\begin{array}{l}
\dot{x}_{1}=x_{2} \\
\vdots \\
\dot{x}_{n}=x_{n+1}+b u \\
\dot{x}_{n+1}=h(t) \\
y=x_{1}
\end{array}\right.
$$

$f$ and $h(t)=\dot{f}$ are assumed unknown. The nonlinear extended state observer described as follows [11]:

$$
\left\{\begin{array}{l}
\dot{z}_{1}=z_{2}+\beta_{1} g(e, a, \delta) \\
\vdots \\
\dot{z}_{n}=z_{n+1}+b u+\beta_{n} g(e, a, \delta) \\
\dot{z}_{n+1}=\beta_{n+1} g(e, a, \delta) \\
e=y-z_{1}
\end{array}\right.
$$

where $z_{1}, z_{2}, \ldots, z_{n}$ are estimated states and $\beta_{1}, \ldots, \beta_{n+1}$ are observer gains, and function $g(e, a, \delta)$ is defined as follows:

$$
g(e, a, \delta)= \begin{cases}|e|^{a} \operatorname{sign}(e) & |e| \succ \delta \\ \frac{e}{\delta^{1-a}} & |e| \leq \delta\end{cases}
$$

$\delta \succ 0$ and $a$ is chosen between 0 and $1, g(e, a, \delta)$ yields high gain when error is small. $\delta$ is a small number used to limit the gain in the neighborhood of origin. Starting with linear gain $g(\cdot)=e$, the pole placement method can be used for the initial design of this observer, before the nonlinearities are added to enhance the performance.

It should be noted that (4) takes the form of the linear ESO, when $a=1$, which is given as:

$$
\left\{\begin{array}{l}
\dot{z}_{1}=z_{2}+\beta_{1} e \\
\vdots \\
\dot{z}_{n}=z_{n+1}+b u+\beta_{n} e \\
\dot{z}_{n+1}=\beta_{n+1} e \\
e=y-z_{1}
\end{array}\right.
$$

In this paper, fault detection is performed by means of the linear ESO.

\subsection{Stability}

Let $e_{i}=x_{i}-z_{i}, \quad i=1, \ldots n+1$ the error equation can be written as:

$$
\dot{e}=A_{e}+B h
$$

$$
A_{e}=A-L C=\left[\begin{array}{ccccc}
-\beta_{1} & 1 & 0 & \cdots & 0 \\
-\beta_{2} & 0 & 0 & \cdots & 0 \\
\vdots & \vdots & & \vdots & \vdots \\
-\beta_{n} & 0 & \cdots & 0 & 1 \\
-\beta_{n+1} & 0 & \cdots & 0 & 0
\end{array}\right], \quad B=\left[\begin{array}{c}
0 \\
0 \\
\vdots \\
0 \\
1
\end{array}\right]
$$

Obviously, the linear ESO is bounded-input bounded-output (BIBO) stable if $h$ is bounded and the parameters $\left(\beta_{1}, \ldots, \beta_{n+1}\right)$ are selected such that (A-LC) is Hurwits.

In this paper, the observer gains are chosen as follows:

$s^{n+1}+\beta_{1} s^{n}+\cdots+\beta_{n+1}=\left(s+\omega_{0}\right)^{n+1}$

where $\omega_{0}$ denotes the bandwidth of the observer.

\section{TWO-TANK SYSTEM}

The mathematical model of the two-tank system has high nonlinearities and is suitable to evaluate the efficiency of the ESO in the context of the fault detection.

A two-tank system [12] as shown in Figure 2 was chosen for this study. The system consists of one pump and two tanks that are connected by small tubes. In this system, the controlled input is the flow rate of pump, and measurable outputs are the water levels of the two tanks. Using the Torricelli's law, the two dynamic equations are:

$\left\{\begin{array}{l}A_{S} \frac{d h_{1}}{d t}=-a_{1} S_{p 1} \operatorname{sign}\left(h_{1-} h_{2}\right) \sqrt{2 g\left|h_{1-} h_{2}\right|}+q_{1} \\ A_{S} \frac{d h_{2}}{d t}=a_{1} S_{p 1} \operatorname{sign}\left(h_{1-} h_{2}\right) \sqrt{2 g\left|h_{1-} h_{2}\right|} \\ -a_{2} S_{p 2} \sqrt{2 g h_{2}}\end{array}\right.$

where $A_{S}$ is the circular cross-sectional area of each tank; $a_{1}$ and $a_{2}$ are flux coefficients; $S_{p 1}$ and $S_{p 2}$ are the circular cross-section area of each tube; $g$ is the gravity acceleration; $q_{1}$ is the flow rate of pump; $q_{2}$ is the outflow from tank $2 ; h_{1}$ and $h_{2}$ are the water level of each tank.

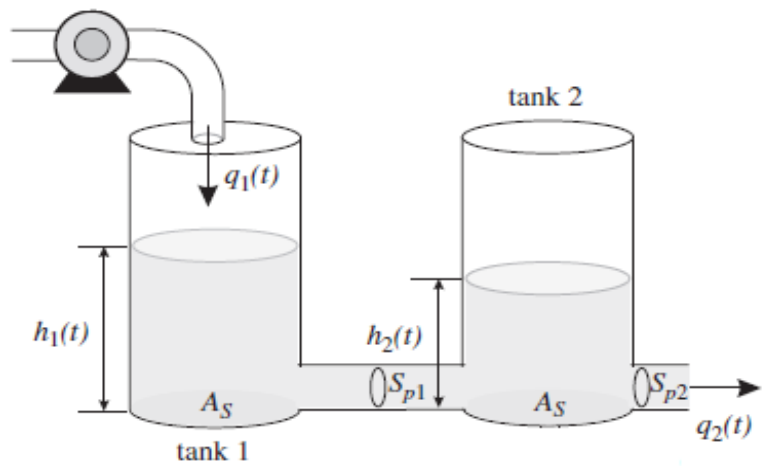

Fig. 2. Diagram of the two-tank system

The ESO formulation for the two-tank model can be rewritten as:

$\left\{\begin{array}{l}\frac{d h_{1}}{d t}=f_{1}+\frac{1}{A_{s}} q_{1} \\ \frac{d h_{2}}{d t}=f_{2}\end{array}\right.$ 
where

$$
\left\{\begin{array}{l}
f_{1}=-\frac{1}{A_{s}}\left[a_{1} S_{p 1} \operatorname{sign}\left(h_{1} h_{2}\right) \sqrt{2 g\left|h_{1}-h_{2}\right|}\right] \\
f_{2}=\frac{1}{A_{s}}\left[\begin{array}{l}
a_{1} S_{p 1} \operatorname{sign}\left(h_{1-} h_{2}\right) \sqrt{2 g\left|h_{1-} h_{2}\right|} \\
-a_{2} S_{p 2} \sqrt{2 g h_{2}}
\end{array}\right]
\end{array}\right.
$$

So we have

$$
\dot{y}(t)=f+b_{0} u
$$

The model of (13) can be written as state space form

$$
\left\{\begin{array}{l}
\dot{x}_{1}=x_{2}+b_{0} u \\
\dot{x}_{2}=h \\
y=x_{1}
\end{array}\right.
$$

where

$$
y=x_{1}=\left[\begin{array}{l}
h_{1} \\
h_{2}
\end{array}\right], u(t)=\left[\begin{array}{c}
q_{1} \\
0
\end{array}\right], x_{2}=\left[\begin{array}{l}
f_{1} \\
f_{2}
\end{array}\right]
$$

The state space observer can be written as

$$
\left\{\begin{array}{l}
\dot{z}=A z+B u+L(y-\hat{y}) \\
\hat{y}=C z
\end{array}\right.
$$

where $\hat{y}=\left[\begin{array}{ll}\hat{h}_{1} & \hat{h}_{2}\end{array}\right]^{T}$ as the estimated output, $z=\left[\begin{array}{ll}z_{1} & z_{2}\end{array}\right]^{T}$ $\left(z_{1}=\left[\begin{array}{ll}z_{11} & z_{12}\end{array}\right]^{T}\right.$ and $z_{2}=\left[\begin{array}{ll}z_{21} & z_{22}\end{array}\right]^{T}$ ).

$\mathrm{L}$ is the observer gain, with a chosen gain, the estimated $\mathrm{z}$ vector can be used to monitor the system outputs and the system dynamics in real time. As time proceeds, $z_{1}$ approaches the system outputs (i.e. $h_{1}$ and $h_{2}$ ), and $z_{2}$ approaches the system dynamics (i.e. $f_{1}$ and $f_{2}$ ).

\section{SENSOR FAULT DETECTION}

The fault detection problem is to indicate whether a fault occurred or not. Traditionaly, faults are considered detected if the abrupt chang of the outputs $\left(\Delta y_{i}\right)$ exceeds the predetermined value [13]. When using the ESO, a fault is considered detected if the abrupt chang of the outputs $\left(\Delta z_{1 i}\right)$ or the abrupt chang of general system dynamics $\left(\Delta z_{2 i}\right)$ exceeds the predetermined value.

For the two-tank system, the appropriate observer bandwidth is chosen as 2 .

The tracking errors for $h_{1}$ and $h_{2}$ is very small, as shown in Figure 4. Interestingly, $z_{21}$ and $z_{22}$ converge to the unknown functions $f_{1}$ and $f_{2}$, as shown in Figure 5.

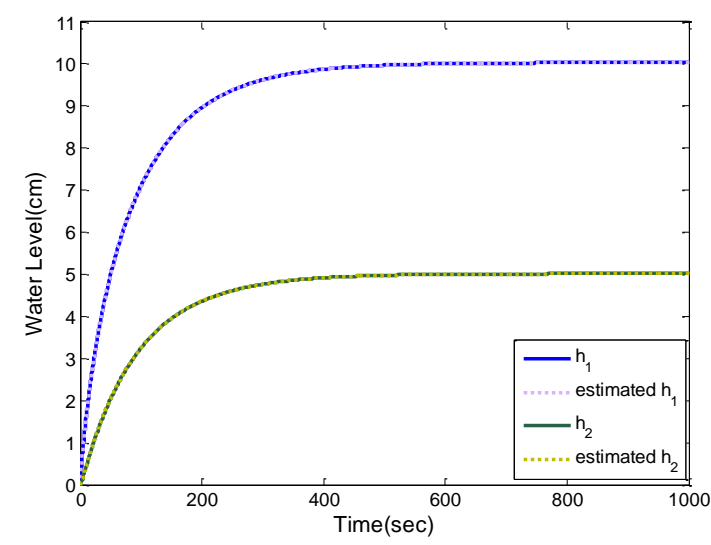

Fig. 3. The curve of the actual and estimated water levels of two-tank system
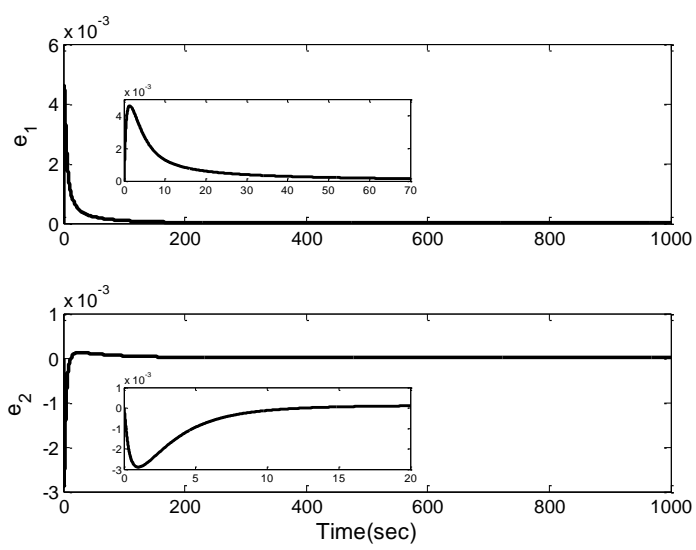

Fig. 4. The observer error
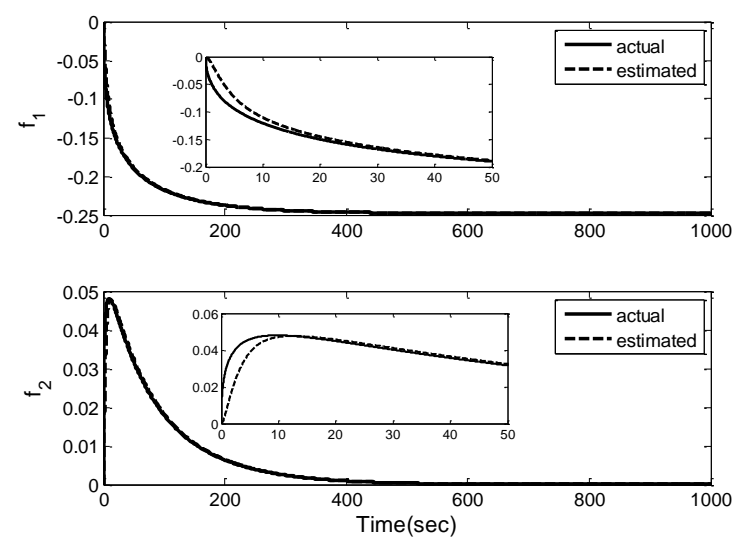

Fig. 5. The curve of the actual and estimated system dynamics of two-tank system

\subsection{Fault detection without noise}

In the simulation example, we assume that a offset fault , $\Delta h_{1}=0.7(\mathrm{~cm})$, in the water level sensor $h_{1}$ occurs at $\mathrm{t}=600 \mathrm{~s}$. 


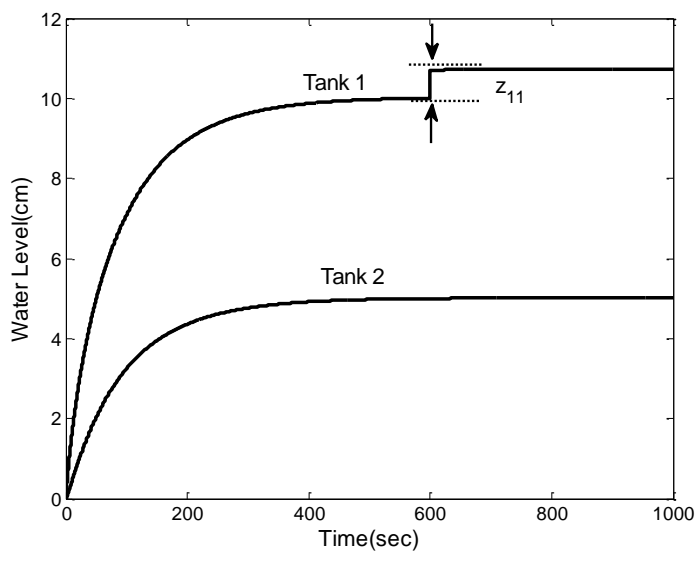

Fig. 6. Estimated water levels of two-tank system for fault detection
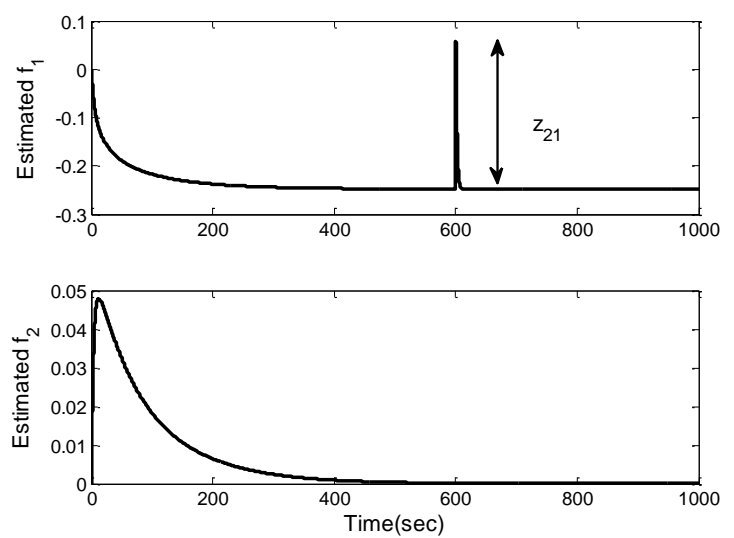

Fig. 7. Estimated system dynamics of two-tank system for fault detection

Figure 6 shows the detected fault based on observing the estimated outputs, $\Delta z_{1 i}$ and Figure 7 shows the detected fault based on observing the estimated system dynamics, $\Delta z_{2 i}$.

\subsection{Fault detection with noise}

Because in the real world noise is inevitable, the output system is corrupted by white noise. The measurement noise is filtered by ESO, as shown in Figure 9.

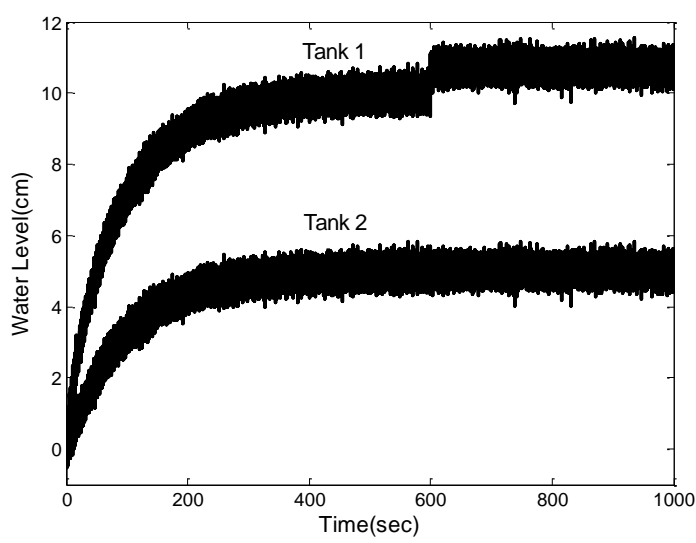

Fig. 8. Measured water levels of two-tank system

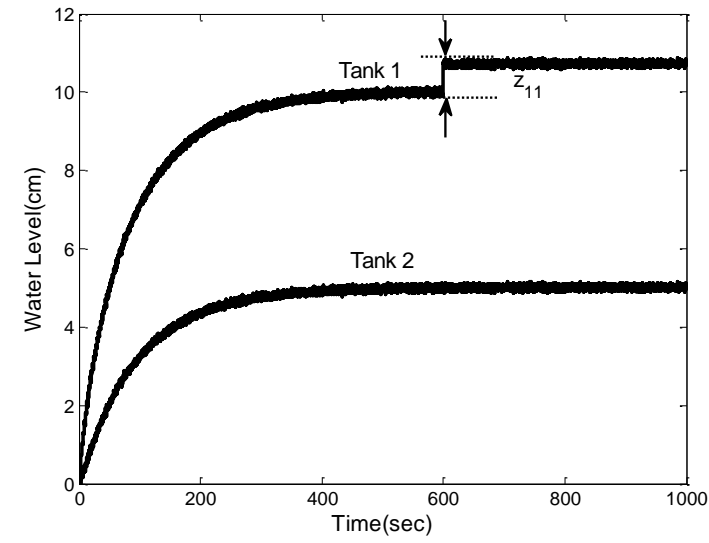

Fig. 9. Estimated water levels of two-tank system for fault detection
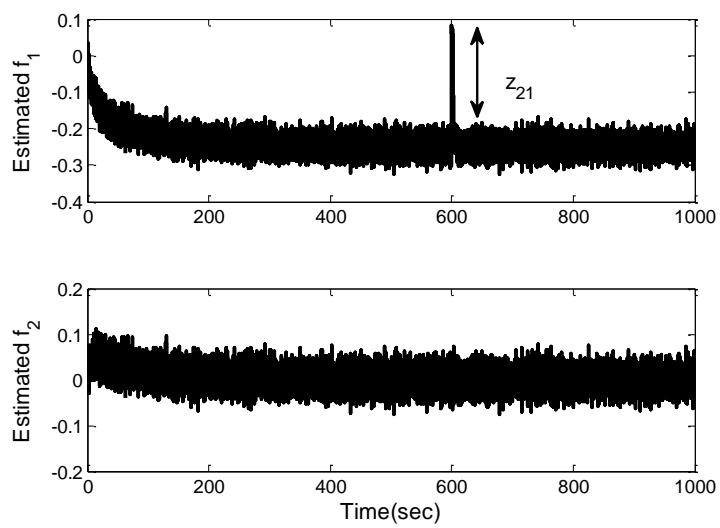

Fig. 10. Estimated system dynamics of two-tank system for fault detection

\section{FAULT ISOLATION}

The fault isolation problem is to determine in which sensor the fault has occurred.

The Sugeno fuzzy system used for fault isolation. The Sugeno fuzzy system structure has two input and two output. The input variables are residuals. According to the fuzzy logic, membership functions are defined for each of the residuals, $R_{h_{1}}$ and $R_{h_{2}}$.

A membership function is a curve that defines how the vector in the input space is mapped to a membership value within 0 and $1[4]$.

Three trapezoid membership functions are applied for the residuals, which are "positive", "zero" and "negative". Figure 11 shows a membership function for the residual $R_{h_{1}}$. The input membership functions for $R_{h_{1}}$ and $R_{h_{2}}$ are the same. The outputs of Sugeno fuzzy system are between 0 and 1 , where "1" represents complete fault.

The five if-then fuzzy rules are made by the rows of Table 1 . For example, the fuzzy rule for a positive offset fault in the water level sensor $h_{1}$ is:

IF $R_{h_{1}}$ positive AND $R_{h_{2}}$ zero THEN $h_{1}+\Delta h_{1}=1$ 
Table 1. Effect of faults on residuals

\begin{tabular}{|lcl|}
\hline Faults & Residuals & \\
\hline & $R_{h_{1}}$ & $R_{h_{2}}$ \\
\hline$h_{1}+\Delta h_{1}$ & + & 0 \\
$h_{1}-\Delta h_{1}$ & - & 0 \\
$h_{2}+\Delta h_{2}$ & 0 & + \\
$h_{2}-\Delta h_{2}$ & 0 & - \\
Normal situation & 0 & 0 \\
\hline
\end{tabular}

$\pm \Delta$, positive or negative offset of sensor signal; + increase; , decrease; 0 no deflection.

In the Table $1, " h \pm \Delta h "$ means that the water level sensor is faulty.

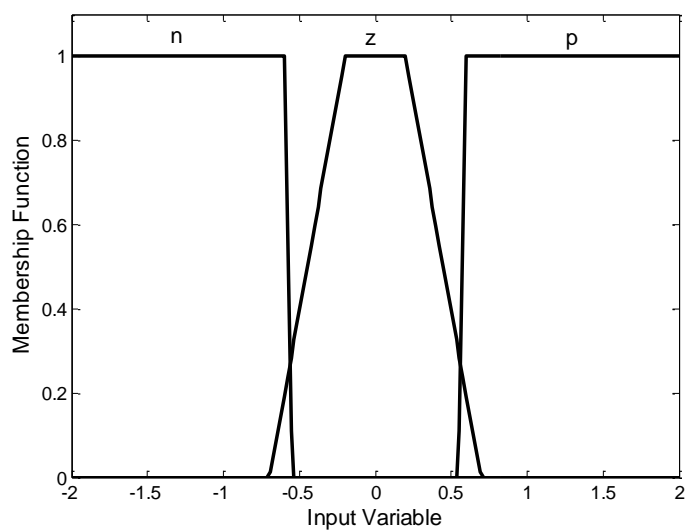

Fig. 11. Membership function for $\boldsymbol{R}_{h_{1}}$
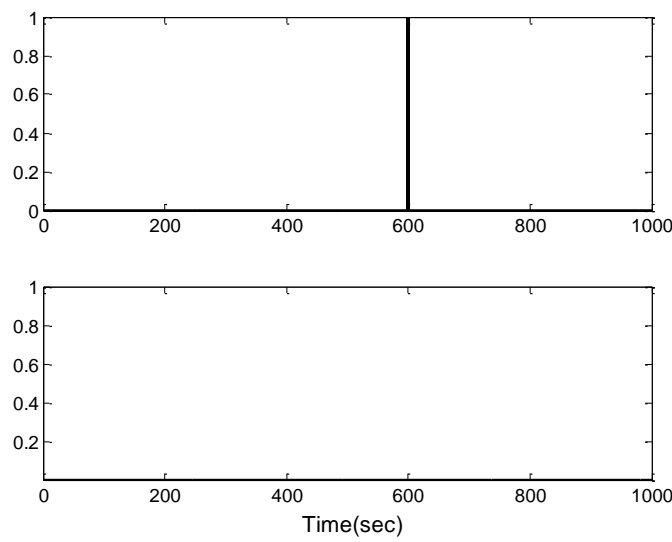

Fig. 12. Result of the fault isolation without noise

Figure 12 shows the result of the fault isolation system in the absence noise.

The range of the measurement noise is [- $-0.90 .9]$. Although, the magnitude of the measurement noise is bigger than the magnitude of the sensor fault, fault isolation is performed successfully. Figure 13 shows the result of the fault isolation system in the presence noise.
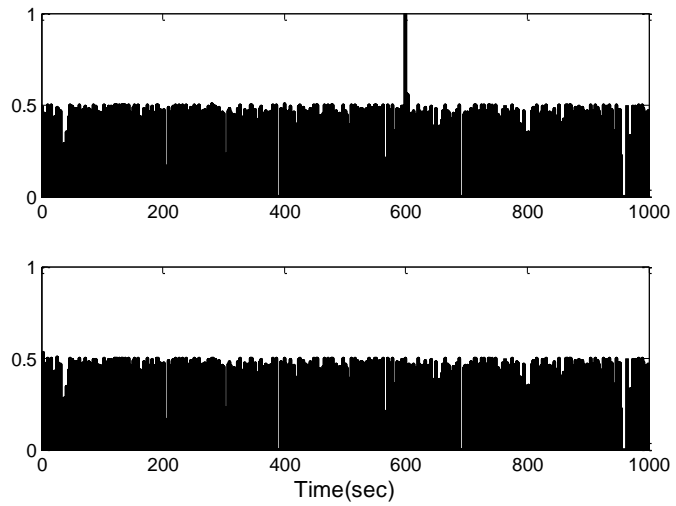

Fig. 13. Result of the fault isolation with noise

\section{CONCLUSION}

This paper proposed a new sensor fault detection technique by using the ESO. The main advantage of ESO is its ability to augment unknown dynamics combined disturbances as an extended state, and estimate it in real time by using inputoutput data. Unlike many other observer, the ESO does not require an accurate mathematical model of the system, therefore, its robustness against uncertainty in the plant dynamics and external disturbance is inherent in its structure.

The fuzzy logic with appropriate fuzzy membership functions, can be applied to evaluate the residuals. By combining the ESO with fuzzy logic, faults could be detected with a good isolation performance.

\section{REFERENCES}

[1] M. Fang, Y. Tian and L. Guo, "Fault diagnosis of nonlinear system based on generalized observer", Applied Mathematics and Computation, vol. 185, pp. 1131-1137, 2007

[2] J. Bokor and Z. Szabo, "Fault detection and isolation in nonlinear systems", Annual Reviews in Control, vol. 33, pp. 113-123, 2009.

[3] V. Venkatasubramanian, R. Rengaswamy, K. Yin and S.N. Kavuri, "A review of process fault detection and fault diagnosis. Part I. Quantitative model-base methods", Computer and Chemical Engineering, vol. 27, pp. 293311, 2003.

[4] D. Ye, C. Zhang and P.P. Lin, " Fault Diagnosis by an Observer-Based Fuzzy Decision System", International Conference on Intelligent Human-Machine Systems and Cybernetics, 2009.

[5] F. Nejjari, V. Puig, L. Giancristofaro and S. Koehler, "Extended Luenberger Observer-Based Fault Detection for an Activated Sludge Process", Proceedings of the $17^{\text {th }}$ World Congress The International Federation of Automatic Control, Seoul, Korea, July 6-11, 2008.

[6] I. Samy, I. Postlethwaite and D-W. Gu, "Survey and application of sensor fault detection and isolation schemes", Control Engineering Practice, vol. 19, pp. 658674, 2011.

[7] J. Zarei and J. Poshtan, "SENSOR FAULT DETECTION AND DIAGNOSIS OF A PROCESS USING UNKNOWN INPUT OBSERVER", Mathematical and 
Computational Application, vol. 16, no. 1, pp. 31-42, 2011.

[8] S. Cao and L. Guo, "Fault Diagnosis with Disturbance Rejection Performance Based on Disturbance Observer", Joint 48th IEEE Conference on Decision and Control and $28^{\text {th }}$ Chinese Control Conference, Shanghai, P.R. China, December 16-18, 2009.

[9] L.F. Mendonca, J.M.C. Sousa and J.M.G. Sada Costa, "An architecture for fault detection and isolation based on fuzzy methods", Expert System with Applications, vol. 36, pp. 1092-1104, 2009.

[10] J. Han "A Class of Extended State Observer for Uncertain Systems", Control and Decision, vol. 10, no. 1 , pp. $85-88,1995$.
[11] X. Tang and Y. Huang. "Capability of Extended State Observer for Estimating Uncertainties", American Control Conference, 10-12 June, 2009.

[12] S. Oblak, I. Škrjanc, and S. Blažič, "Fault detection for nonlinear systems with uncertain parameters based on the interval fuzzy model", Engineering Application of Artificial Intelligence, vol. 20, pp. 503-510, 2007.

[13] R. Liang, P.P. Lin and D. Ye, "Fault Detection Based on the Estimated Parameter of System Model", International Conference on Measuring Technology and Mechatronics Automation, 2010. 